\title{
AVALIAÇÃO DO ESCOAMENTO E RADIOPACIDADE DE DOIS CIMENTOS ENDODÔNTICOS
}

\author{
EVALUATION OF FLOW AND RADIOPACITY OF TWO ROOT CANAL \\ SEALERS
}

\author{
Luciana Azevedo Curty Andinós* \\ Izabel Coelho Gomes Camões" \\ Lílian Ferreira Freitas"** \\ Cristina Nunes Santiago \\ Cinthya Cristina Gomes
}

\begin{abstract}
RESUMO
Introdução: O objetivo deste estudo é avaliar as propriedades do escoamento e radiopacidade de dois cimentos endodônticos, Endofill e EZ-Fill de acordo com a especificação número 57 da ADA. Método: Para análise do escoamento foram feitas 20 amostras divididas em GRUPO 1 (Endofill) e GRUPO 2 (EZ-Fill). Para tal 0,5 $\mathrm{ml}$ do cimento era depositado no centro de uma placa de vidro de $10 \times 10 \mathrm{~cm}$. Decorridos 3 minutos, outra placa de vidro e um peso de chumbo, totalizando $120 \mathrm{~g}$, foram colocados sobre o material. Após 10 min o peso foi removido e a média do diâmetro maior e menor dos discos obtidos foi determinada por um paquímetro. Para a análise da radiopacidade 10 anéis metálicos foram utilizados: 5 do Grupo 3 (EZ-Fill) e 5 do Grupo 4 (Endofill) foram preenchidos com os cimentos. Os corpos de prova foram radiografados com uma escala de alumínio com 10 degraus uniformes. Para a obtenção das radiografias foi utilizado um aparelho de raio $X$ Dabi Atlante a uma distância foco objeto de $400 \mathrm{~mm}$ e tempo de exposição 0,1 seg. As radiografias foram digitalizadas, armazenadas em um microcomputador e analisadas através de um software específico. Os dados foram submetidos ao teste $t$ de Student a 5\% apresentando diferença significante $(\mathrm{p}<0,0001)$. Resultados: Os resultados encontrados demonstraram escoamento superior a $25 \mathrm{~mm}$ em ambos os cimentos dos grupos 1 e 2 e radiopacidade superior a $3 \mathrm{~mm}$ de alumínio dos grupos 3 e 4, cumprindo-se a especificação número 57 da ADA. Conclusão: O cimento Endofill apresentou escoamento superior, enquanto o cimento EZ-Fill apresentou maior radiopacidade.

DESCRITORES: Cimentos dentinários $\bullet$ Escoamento $\bullet$ Raios- $X \bullet$ Radiografia $\bullet$ Digitalis
\end{abstract}

\section{ABSTRACT}

Introduction: The purpose of this study is to evaluate the flow properties and radiopacity of two root canal sealers filling, the Endofill (Grossman of cement) and EZ-Fill according to ADA specification number 57 for filling materials). Methods: 20 samples were divided into two groups: Group 1 (Endofill), Group 2 (EZ-Fill). Each group was composed of 10 samples. For the analysis of flow $0.5 \mathrm{ml}$ of material was loaded into a disposable syringe and deposited in the center of a placar glass $10 \mathrm{~cm}$ X $10 \mathrm{~cm}$. 180 seconds after the start of operation, another plate of glass and a weight of lead were placed over the material, totaling $120 \mathrm{~g} .10 \mathrm{~min}$ utes after the beginning of the mixture the weight was removed and the average diameter of the largest and smallest of the disc obtained was determined by a caliper. For the analysis of radiopacity, 10 metal rings, 5 of Group 3 (EZ-Fill) and 5 of Group 4 (Endofill) containing holes of $2 \mathrm{~mm}$ in height and $10 \mathrm{~mm}$ in diameter were filled with cement. The samples were radiographed together with a ranger of $99 \%$ aluminum (alloy 1100 ) with 10 uniform steps ( $1 \mathrm{~mm}$ to $10 \mathrm{~mm}$ thick). To obtain the radiographs an X-ray apparatus Dabi Atlante (70 kvp, $8 \mathrm{~mA}$ ) was used to a focus object distance of $400 \mathrm{~mm}$ and exposure time 0.1 seconds. The radiographs were digitized using a Kodak scanner - LS 85 Film Digitizer, USA, stored in a microcomputer and analyzed by a specific software for reading images (Image J). The data were submitted to the Student test at $5 \%$ showing a significant difference $(\mathrm{p}<0.0001)$. Results: The results obtained were: flow higher than $25 \mathrm{~mm}$ in both cements of Group 1 (Endofill $54.69 \mathrm{~mm}$ ) and Group 2 (EZ-Fill $42.41 \mathrm{~mm}$ ) and radiopacity of Group 3 (EZ-Fill) equivalent to $10 \mathrm{~mm}$ of aluminum and Group 4 (Endofill) equivalent to $7 \mathrm{~mm}$ aluminum. It was concluded that the cement Endofill showed a higher flow than the cement EZ-Fill and the EZ-Fill cement presented a higher radiopacity of the cement Endofill.

DESCRIPTORS: Dental cements • Root canal therapy • Endodontics

\footnotetext{
* Cirurgiã Dentista /e-mail: lucurty@yahoo.com.br Universidade Federal Fluminense - UFF

** Professora Doutora e Coordenadora do Curso de Especialização em Endodontia/ Universidade Federal Fluminense - UFF e-mail: iccamoes@gmail.com

*** Professora Doutora e Vice-coordenadora do Curso de Especialização em Endodontia/Universidade Federal Fluminense - UFF e-mail: lilianffreitas@uol. com.br

**** Professora Mestre do Curso de Especialização em Endodontia/Universidade Federal Fluminense - UFF e-mail: cris.santiago@yahoo.com.br

***** Professora Doutora do Curso de Especialização em Endodontia/Universidade Federal Fluminense - UFF e-mail: cigomez@terra.com.br
} 
ANDINÓS LAC

CAMÕES ICG

FREITAS LF

SANTIAGO CN

GOMES CC

AVALIAÇÃO DO

ESCOAMENTO E

RADIOPACIDADE

DE DOIS

CIMENTOS

ENDODONTICOS

16

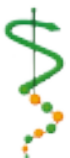

REVISTA DE

ODONTOLOGIA DA

UNIVERSIDADE

CIDADE DE SÃO

PAULO

$2011 ; 23(1): 15-$

22, JAN-ABR

\section{IN TRO DUÇÃ 0}

Para alcançar o sucesso do tratamento endodôntico faz-se necessário o restabelecimento da integridade dos tecidos perirradiculares e preservação da estrutura dentária, proporcionado pelo selamento apical do dente, pelo fechamento dos espaços entre cone de guta percha e parede dentinária e o selamento coronário. Estes objetivam o selamento tridimensional do canal, eliminando vias de recontaminação (Almeida $\left.{ }^{1}, 2005\right)$.

O objetivo da obturação do sistema de canais radiculares é o total preenchimento dos espaços vazios no interior do dente, os quais foram gerados através do processo de sanificação dos canais (Soares e Goldberg², 2001).

Como auxiliadores e verificadores da exatidão da obturação, ainda hoje utilizase a imagem radiográfica. É o único elemento que se dispõe para verificação do selamento hermético do canal. Radiograficamente a obturação tende o mais próximo possível do CDC, sem extravasamento excessivo ou falta de material obturador, na presença de um canal patente (Oliveira et al. ${ }^{3}, 2001$ ).

O material escolhido para a obturação do sistema de canais radiculares é a guta percha, utilizada juntamente com um cimento. Ambos são os que melhor se adaptam aos critérios exigidos e, ainda assim, não fornecem rotineiramente um selamento impermeável ao sistema de canais radiculares, que acabam sofrendo infiltrações em maior ou menor extensão. (Cohen e Burns $\left.{ }^{4}, 2000\right)$.

Um bom cimento endodôntico deve ter as seguintes características: ser biocompatível, bem tolerado pelos tecidos perirradiculares, preencher irregularidades do canal, auxiliar no controle bacteriano, auxiliar no assentamento do principal material obturador, ter sua adesividade aumentada diante da remoção da smear layer e melhorar a possibilidade de obtenção de um selamento impermeável. Se o cimento tiver baixa viscosidade, terá bom escoamento, porque preenche irregularidades do canal e espaços entre materiais e parede dentinária. O bom escoamento, aliado a ação antimicrobiana, melhora a capacidade de descontaminação do canal, eliminando micro organismos presentes em áreas não alcançadas pela instrumentação. (Siqueira et al. ${ }^{5}, 2000$ ).

Novos cimentos e novas técnicas de obturação estão sendo pesquisados na tentativa de se conseguir o desejável selamento apical. As novas tendências dos cimentos estão relacionadas à penetração nos túbulos dentinários abertos, à neutralização ou destruição de microrganismos e seus subprodutos, à união das fases orgânica e inorgânica da dentina, ao fortalecimento do sistema radicular e à indução de resposta cementária regenerativa. (Estrela $\left.{ }^{6}, 2004\right)$.

O presente estudo preocupou-se em comparar o escoamento e a radiopacidade de dois cimentos endodônticos, utilizando como referência a especificação número 57 da Association ${ }^{7}$, (1983).

\section{MÉTODOS}

\section{Escoamento:}

O escoamento dos cimentos endodônticos Endofill (Dentsply - Herpo, Indústria e Comércio, Rio de Janeiro, RJ, Brasil) e EZ Fill (EDS - Essential Dental Systems, USA) foi testado, utilizando-se o método da espalmabilidade ou extensibilidade de acordo com a Especificação n 57 da Association ${ }^{7}$, (1983). Esse método refere-se a capacidade de espalmar ou de tornar plana à superfície de uma preparação quando submetida a uma força, como, também, à facilidade com que essa se espalha e se estende mediante pressão.

Foram pesados 3 gramas de cimento em balança de precisão (Shimadzu do Brasil Comércio Ltda, Ax200, 2006, Classe I, portaria INMETRO 065-2003) e em seguida mediu-se $0,20 \mathrm{ml}$ do líquido. Cada cimento foi espatulado seguindo a orientação dos fabricantes, até conseguir-se a consistência clínica ideal. Então, 0,5ml de cada cimento foi captado com o auxilio da seringa descartável (BD - Becton Dickison) e depositado no centro da placa de vidro de dimensões $10 \times 10 \mathrm{~cm}$ (Vidraçaria Ritz). Após decorridos aproximadamente 3 minutos do início da espatulação, colocou-se centralmente por sobre o cimento obturador um conjunto composto por outra placa de vidro e carga adicional que consiste em peso de chumbo, (casa 
de material de pesca) totalizando 120 gramas. Removeu-se o peso adicional após 10 minutos do início da mistura.

Foram realizadas dez amostras de cada cimento totalizando 20 amostras. Foram divididas em 2 grupos: Grupo 1 - cimento Endofill e Grupo 2 - cimento EZ Fill.

Para medição do comprimento dos discos obtidos através do escoamento dos cimentos endodônticos foi utilizado o método convencional recomendado pela ADA, em que o maior e o menor comprimento do disco formado é medido, com o auxílio de um paquímetro digital (MITUTOYO Sul Americana Ltda).

\section{Radiopacidade}

Para a realização da avaliação da radiopacidade dos cimentos endodônticos estudados, foi seguida a especificação $\mathrm{n}^{\circ}$ 57 da Association ${ }^{7}$, (1983). Foi utilizado um anel metálico de $10 \mathrm{~mm}$ (Serralheria Iguatemi - Rio de Janeiro/RJ) de diâmetro interno por $2 \mathrm{~mm}$ de altura. Foram realizadas 5 amostras por grupo, totalizando 10 amostras divididas em 2 grupos: Grupo 3 - Cimento EZ-Fill e Grupo 4 - Cimento Endofill, que foram manipulados de acordo com a orientação dos seus respectivos fabricantes. Os cimentos foram compactados em cada anel e colocados sobre uma placa de vidro (JON Comércio de produtos Odontológicos Ltda, São Paulo, SP, Brasil), havendo, entre eles, uma folha de papel celofane (Cromus - Mauá/SP), foram colocadas sobre o cimento outra folha de papel celofane e outra placa de vidro. Assim os cimentos foram deixados até endurecerem.

Uma escala de alumínio de espessura variável (1 a $10 \mathrm{~mm}$ ) foi utilizada como referência para medir a radiopacidade, possibilitando a comparação das espessuras da amostra com a escala de alumínio após as tomadas radiográficas.

As radiografias foram obtidas através do aparelho Dabi Atlante Spectro, 70 Kvp, $9 \mathrm{~mA}$, em filme radiográfico periapical (Kodak Dental Brasil) e oclusal (Kodak Dental Brasil), com os anéis de cimento e a cunha metálica em filmes radiográficos separados. Os cimentos foram agrupados de 5 em 5 anéis para serem radiografados (Grupos 3 e 4) a uma distância foco-filme de $400 \mathrm{~mm}$ e um tempo de exposição de
1 segundo. Foram feitas três radiografias para cada grupo com 5 amostras, (três radiografias para o Grupo 3 e três para o Grupo 4).

Os filmes foram processados manualmente: 2 minutos de revelação (Kodak Dental brasileiro - CAT 1562826), 30 segundos de enxágue e 5 minutos de fixação (Kodak Dental brasileiro - CAT 8610248) e lavagem durante 10 minutos em água corrente. E deixados secar espontaneamente. As radiografias do material e da cunha foram digitalizadas através do aparelho Kodak LS 85 Film digitizer USA - número de série A0024 - Laboratório de Ciências Radiológicas - UERJ (digitalização de 3000 pixels por linha), armazenadas em um microcomputador (Laboratório de Ciências Radiológicas - UERJ) e posteriormente foi realizada a leitura através de um programa de computador próprio (Image J, Laboratório de Ciências Radiológicas - UERJ). Assim, foi determinada a espessura de alumínio equivalente ao corpo de prova de cada cimento avaliado.

A medição da radiopacidade para cada grupo das amostras dos cimentos e cada milímetro da escala de alumínio foi realizada em três pontos diferentes. Foram obtidas três medidas de opacidade em cada anel de cimento, nas três radiografias, em pontos diferentes, para obter-se a média de opacidade de cada grupo. A média de opacidade foi obtida a partir da soma e divisão dos valores de opacidade dos 5 anéis de cada amostra de radiografia, para cada cimento diferente, totalizando 10 anéis.

\section{3 - RESULTADOS}

\section{Escoamento}

Os resultados obtidos foram discos com diâmetros superiores a $25 \mathrm{~mm}$, correspondendo à recomendação da especificação número 57 da Association ${ }^{7}$, (1983). De acordo com a metodologia aplicada, a análise dos dados mostra que o maior halo no sentido vertical e horizontal foi alcançado pelo cimento Endofill.

Os valores dos halos formados pelo escoamento do cimento Endofill (Grupo 1) pode ser observado no Gráfico 1 e os valores do EZ fill (Grupo 2) no 2. A média aritmética entre os valores horizontal e vertical de cada halo formado para os dois
ANDINÓS LAC

CAMÕES ICG

FREITAS LF

SANTIAGO CN

GOMES CC

AVALIAÇÃO DO

ESCOAMENTO E

RADIOPACIDADE

DE DOIS

CIMENTOS

ENDODONTICOS
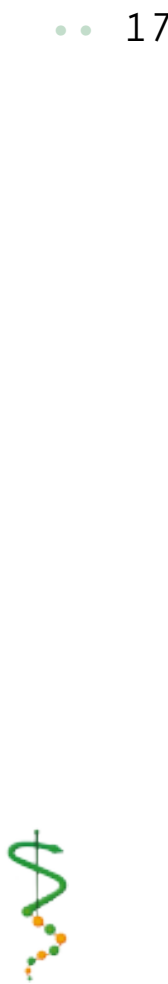

REVISTA DE ODONTOLOGIA DA UN I VERS I DADE Cidade de SÃo PAULO

2011; 23(1): 15 22, JAN-ABR 
ANDINÓS LAC

CAMÕES ICG

FREITAS LF

SANTIAGO CN

GOMES CC

AVALIAÇÃO DO

ESCOAMENTO E

RADIOPACIDADE

$D E$ DOIS

CIMENTOS

ENDODONTICOS

GRÁFICO 1 - Valores ( $\mathrm{mm}$ ) dos halos formados pelo escoamento do cimento Endofill.

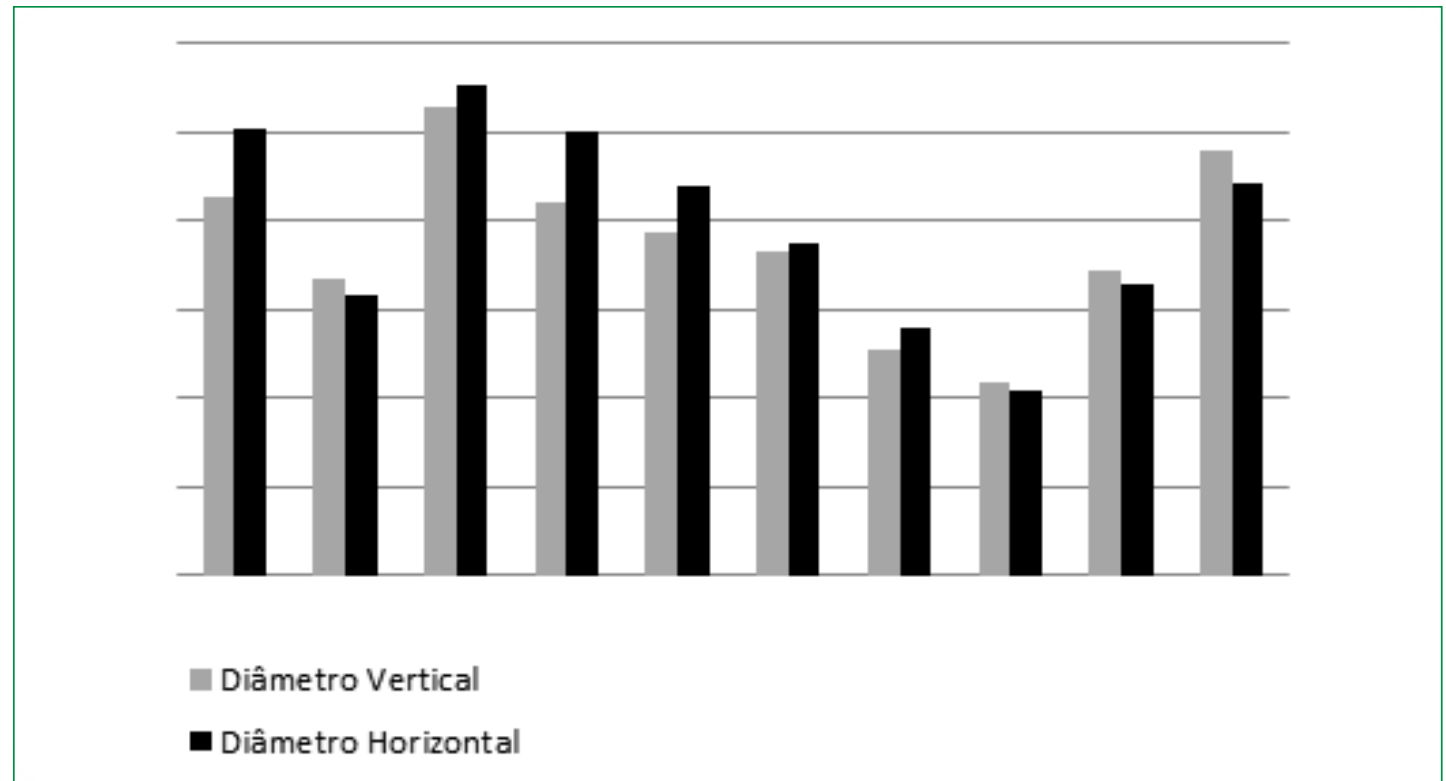

GRÁFICO 2 - Valores ( $\mathrm{mm}$ ) dos halos formados pelo escoamento do cimento EZ-fill.

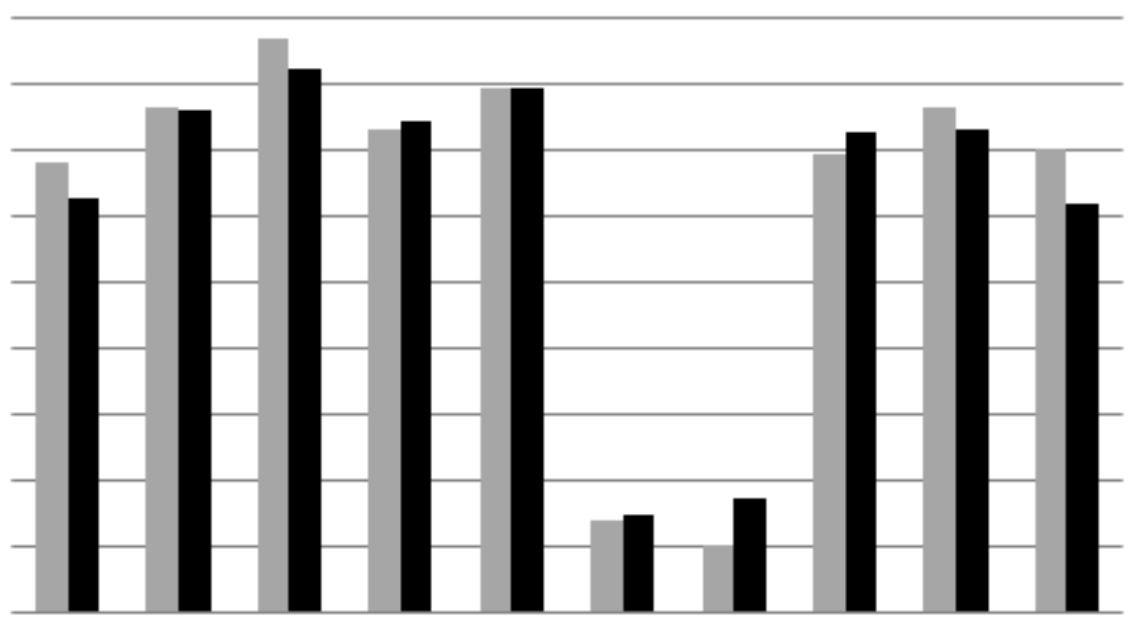

Diâmetro Vertical

Diâmetro Horizontal

grupos podem ser avaliados no Gráfico 3.

Os dados do gráfico acima foram analisados estatisticamente pelo teste $\mathrm{t}$ de Student a $5 \%$, que revelou que existe diferença estatisticamente significante entre o escoamento dos dois tipos de cimentos $(\mathrm{P}<0,0001)$. Como pode ser observado, o escoamento do cimento Endofill (Grupo 1) foi superior ao EZ Fill em todas as amostras.

\section{Radiopacidade}

Os resultados obtidos através da leitura, realizada pelo programa de computador, foram correspondentes a 0,83 de opacidade para o Grupo 3 (cimento EZ-Fill) equivalente a $10 \mathrm{~mm}$ de alumínio e 1,15 de opacidade para o Grupo 4 (cimento Endofill) equivalente aproximadamente a $7 \mathrm{~mm}$ de alumínio, correspondendo à recomendação da especificação número 57 da Association ${ }^{7}$, (1983) que determina que um cimento não deve apresentar radiopacidade inferior equivalente a $3 \mathrm{~mm}$ de alumínio. De acordo com a metodologia aplicada, a análise dos dados mostra que o material mais radiopaco foi o cimento EZ-Fill - Grupo 3.

Cada grupo de 5 anéis foi radiografado três vezes (imagens 1, 2 e 3) e estão representados pelas amostras I, II e III para 


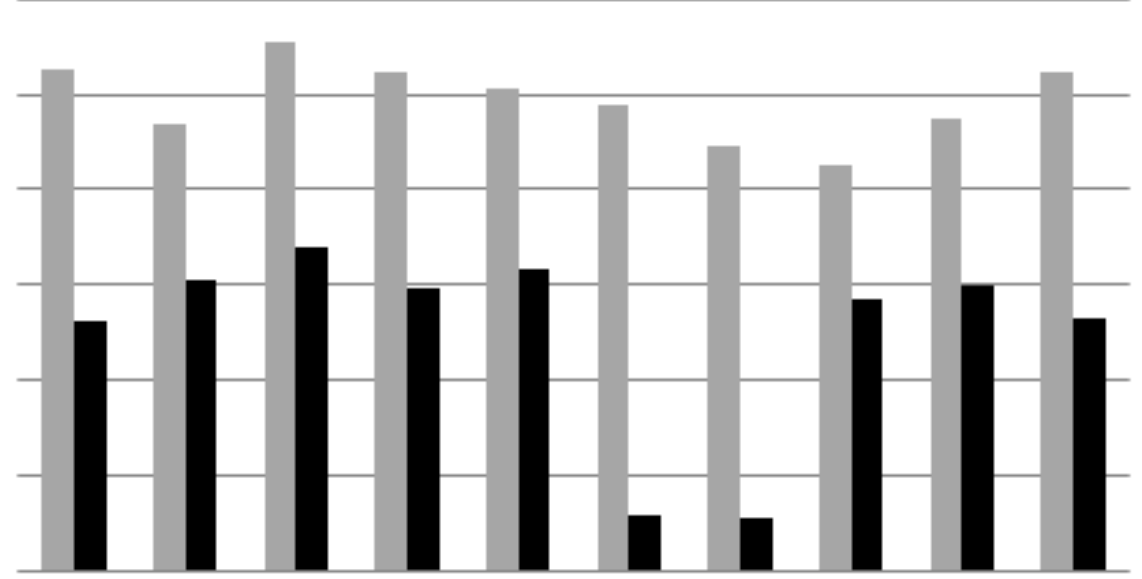

FREITAS LF

SANTIAGO CN

GOMES CC

AVALIAÇÃO DO

ESCOAMENTO E

RADIOPACIDADE

DE DOIS

CIMENTOS

ENDODONTICOS

Cimento End ofill

- Cimento EZ Fill

GRÁFICO 4 - Valores ( $m$ m de alumínio) da média aritmética da radiopacidade dos cimentos Endofill e EZ-fill (grupo 3 e 4).
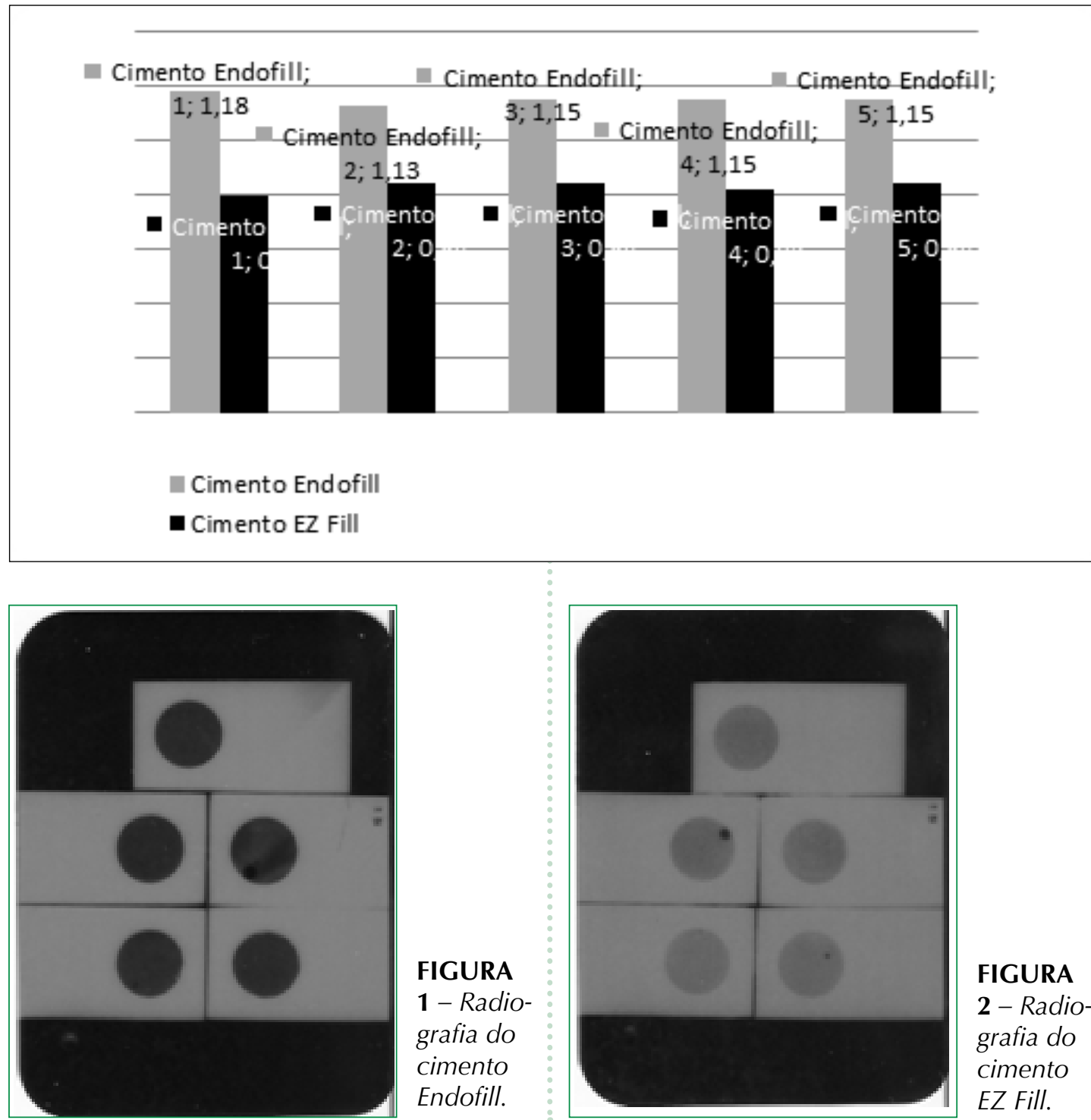

FIGURA 1 - Radiografia do cimento Endofill.

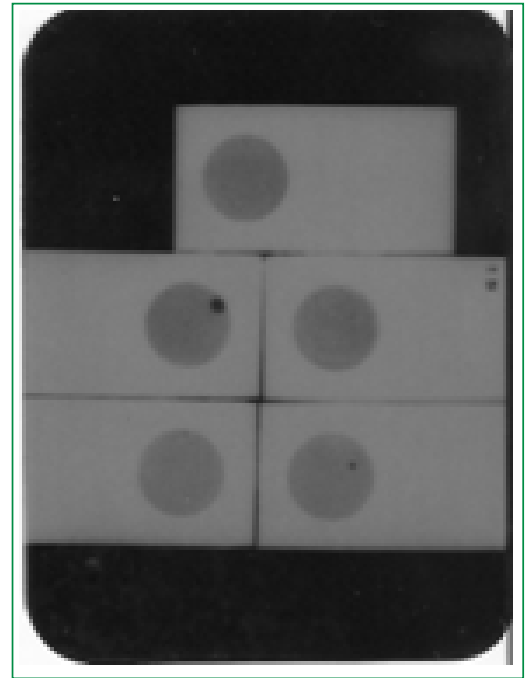

FIGURA

2 - Radiografia do cimento EZ Fill.

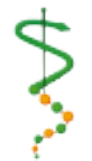

REVISTA DE

ODONTOLOGIA DA UN I VERS I DADE

CIDADE DE SÃO PAULO

2011; 23(1): 15

22 , JAN-ABR 


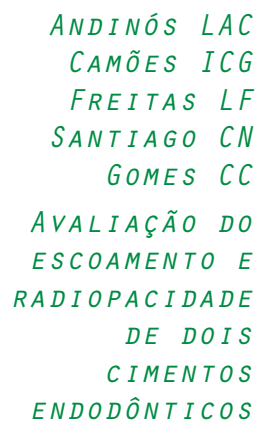

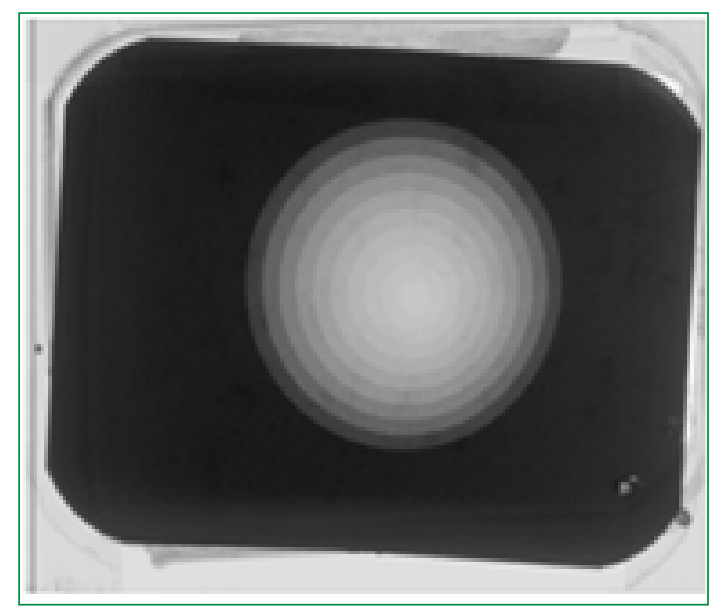

FIGURA 3 - Radiografia da cunha.

o Grupo 3 (cimento EZ-Fill) e IV, V e VI para o Grupo 4 (cimento Endofill). A soma total dos valores do cimento EZ-Fill é de 4,14 e a sua média aritmética é de 0,83 de opacidade e a soma total dos valores do cimento Endofill é de 5,76 e a média aritmética é de 1,15 de opacidade.

No Gráfico 4, observam-se os valores finais das médias aritméticas de cada cimento e sua equivalência em milímetros de alumínio.

Os dados foram analisados pelo teste $\mathrm{t}$ de Student a 5\% que revelou diferença estatisticamente significante entre a opacidade dos dois tipos de cimento $(P<0,0001)$.

\section{I SCUSSÃO}

A propriedade do escoamento é um fator determinante no sucesso de uma obturação hermética do sistema de canais radiculares e, assim o presente estudo elegeu esta metodologia também utilizada por Siqueira et al. ${ }^{5}$ (2000) e Savioli et al. ${ }^{8}$ (1994).

A radiopacidade é também uma propriedade física indispensável aos cimentos endodônticos, pois permite a visualização e avaliação radiográfica da obturação do sistema de canais radiculares, sendo o método clínico mais utilizado. O presente estudo optou por raio $\mathrm{X}$ convencional $\mathrm{e}$ não pelo digital, por ser aquele o método mais utilizado pelos cirurgiões-dentistas e que representa um menor custo.

A determinação do escoamento do cimento pode ser realizada por muitas técnicas como viscosidade, penetrabilidade, espalmabilidade e extrusão. O método utilizado neste trabalho foi a espalmabilidade, seguindo orientações e normas internacionais da especificação número 57 da $\operatorname{ADA}^{7}$ (1983). A recomendação é que o disco formado pelo cimento deve ter no mínimo 25 mm de diâmetro.

De acordo com os resultados encontrados nesta pesquisa, o Grupo 1 - Endofill

GRÁFICO 5 - Equivalência em milímetros de alumínio dos cimentos: grupos 3 e 4 - EZ-Fill e Endofill.

\section{RADIOPACIDADE DOS CIMENTOS}

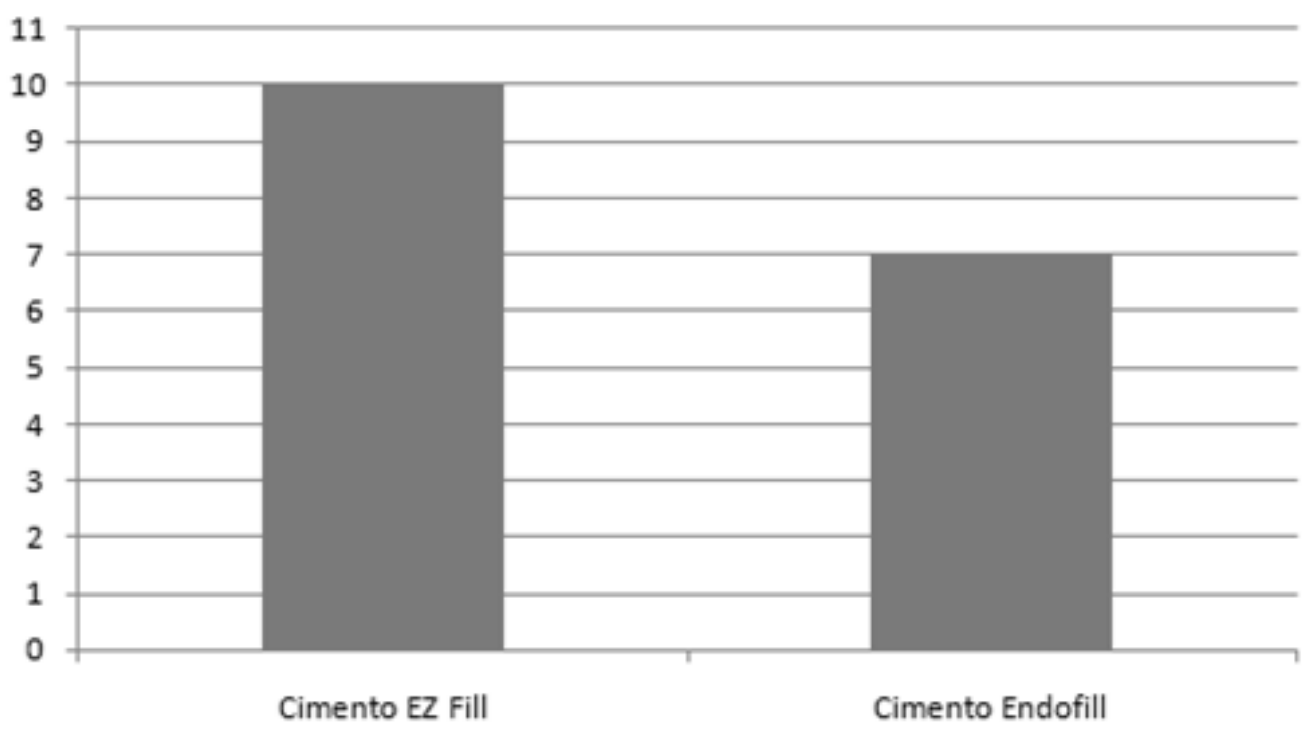


obteve um escoamento significativamente superior ao Grupo 2 - EZ Fill. O estudo realizado por Guimarães et al. ${ }^{9},(1999)$ avaliou o escoamento de seis cimentos, comparou o Endofill, Sealer 26, N-Rickert, Ketac-Endo, Sealapex e Pulp Canal EWT. Os autores também demonstraram que o Endofill tem um bom escomento, pois concluíram que o maior escoamento apresentado foi do Pulp Canal EWT, seguido do Endofill, Sealer 26 e N-Rickert e que os cimentos Ketac Endo e Sealapex não apresentaram escoamento algum.

Para a determinação da radiopacidade dos cimentos foi ultilizado um método padronizado para medir, através de radiografias, a radiopacidade de cimentos endodônticos e compara-los de acordo com normas internacionais da especificação 57 da Association$^{7}$, (1983) para materiais obturadores de canais radiculares, onde o cimento deve ter opacidade de, no mínimo, o equivalente a $3 \mathrm{~mm}$ de alumínio. Essa metodologia foi utilizada por outros autores, entre eles Elias e Costa ${ }^{10}, 2003$; Costa et al. ${ }^{11}, 2002$; Baksi et al. ${ }^{12}, 2008$; Tasdemir et al. ${ }^{13}, 2008$; entre outros. Foram analisados dois grupos de cimentos, Grupo 3 (cimento EZ-Fill) e Grupo 4 (cimento Endofill)., A escala de alumínio, em 10 degraus, foi escolhida como padrão de referência, pois permite a comparação das espessuras das amostras com a escala em radiografias, como foi observado por Costa et al. ${ }^{11}, 2002$, que pesquisaram e encontraram espessuras de equivalência em mm de alumínio de quatro cimentos endodônticos diferentes: Pulp Canal Sealer - 14,6 mm; Tubliseal - 10,3 mm; $\mathrm{AH}$ Plus - 14,7 mm e Sealapex - 5,7 mm. Oliveira et al. ${ }^{3}, 2001$, também pesquisaram a radiopacidade de três diferentes cimentos: Sealapex, Sealer 26 e Endofill e chegaram à conclusão de que o cimento Endofill apresentou radiopacidade superior aos outros, seguido do cimento Sealer 26 e do Sealapex. Elias e Costa ${ }^{10}, 2003$, estudaram a radiopacidade dos cimentos endodônticos Endofill e Sealer 26 e concluíram que o cimento Endofill apresentou maior radiopacidade (10 mm de alumínio) que o cimento Sealer 26. Os dados encontrados ratificam os encontrados neste estudo.

A análise da radiopacidade pode ser realizada por duas metodologias: através do aparelho fotodensitômetro, o qual é utilizado para registrar a leitura das imagens radiográficas dos cimentos, e através da utilização da radiografia digital. Nesta pesquisa, as radiografias foram digitalizadas, em um computador e analisadas em um programa que traduz a imagem em valores de tons de cinza da intensidade pixel, o qual permitiu que uma análise mais detalhada da imagem fosse feita.

Baksi et al. ${ }^{12}$ (2008), pesquisaram as diferenças em valor equivalente de alumínio de cimentos endodônticos. Os autores compararam a radiopacidade dos cimentos Acroseal, Diaket, Guttaflow, Pulp Canal Sealer e Roekoseal através da radiografia digital e convencional. A densidade óptica dos cimentos foi avaliada através da transmissão densitométrica e esta traduzida em valores de cinza, determinando a análise digital. Concluíram que as medições dos valores de equivalência de alumínio digital são inferiores aos correspondentes aos valores densitométricos (medidos através de um aparelho densitométrico), considerando que não houve diferença estatística significante para a avaliação dos cimentos.

Tasdemir et al. ${ }^{13}$ (2008) avaliaram a radiopacidade de novos cimentos endodônticos por radiografia digital e as equivalências de alumínio encontradas foram: AH Plus - 10,41; Epiphany - 8,20; Diaket - 6.50; Guttaflow - 4,90 e Adseal - 3,09.

Os cimentos utilizados nesta pesquisa foram adquiridos em lojas de venda de materiais odontológicos, ou seja, tomouse cuidado para que fossem utilizados sem influência dos fabricantes.

Os relatos existentes na literatura sobre a radiopacidade do cimento EZ-Fil são muito escassos, devido, provavelmente, ao fato de se tratar de um novo cimento obturador de canal. Neste estudo a radiopacidade do EZ-Fill foi equivalente a 10 mm de alumínio e a do Endofill equivalente a 7 mm de alumínio.

Em relação às propriedades de escoamento e radiopacidade, os dois cimentos estudados cumpriram as especificações da ADA. Por ser o EZFill um cimento novo, outras pesquisas avaliando e comparando as propriedades desse cimento obturador devem ser realizadas.
ANDINÓS LAC

CAMÕES ICG

FREITAS LF

SANTIAGO CN

GOMES CC

AVALIAÇÃO DO

ESCOAMENTO E

RADIOPACIDADE

DE DOIS

CIMENTOS

ENDODONTICOS
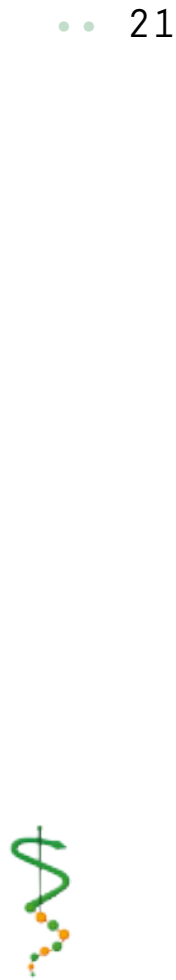

REVISTA DE ODONTOLOGIA DA UN I VERS I DADE Cidade de São PAULO

2011; 23(1): 15 22, JAN-ABR 
ANDINÓS LAC

CAMÕES ICG

FREITAS LF

SANTIAGO CN

GOMES CC

AVALIAÇÃO DO

ESCOAMENTO E

RADIOPACIDADE

DE DOIS

CIMENTOS

ENDODONTICOS

\section{CONCLUSÃO}

Com base nos resultados obtidos e na metodologia empregada, pode-se concluir que:

1. Tanto o cimento Endofill quanto o EZ-Fill apresentaram discos de escoamento superior a $25 \mathrm{~mm}$, cumprindo a Especificação número 57 da Association ${ }^{7}$ (1983).

2. O cimento Endofill apresentou escoamento superior ao cimento EZFill, levando-se em consideração os resultados obtidos através das mé- dias aritméticas das amostras e das análises estatísticas.

3. O cimento EZ-Fill apresentou radiopacidade superior ao cimento Endofill, levando-se em consideração os resultados obtidos através das médias aritméticas das amostras e das análises estatísticas.

4. Tanto o cimento Endofill quanto o EZ-Fill apresentaram radiopacidade superior a $3 \mathrm{~mm}$ de alumínio, cumprindo a determinação da Especificação número 57 da Association ${ }^{7}$ (1983).

\section{REFERÊNCIAS:}

1. Almeida J. Avaliação de diferentes cimentos endodônticos quanto ao escoamento, obturação e selamento marginal em canais laterais artificialmente produzidos em dentes humanos [Mestrado]. Piracicaba: Universidade Estadual de Campinas; 2005.

2. Soares I, Goldberg F. Endodontia: tecnica e fundamentos. Porto Alegre: Artmed; 2001.

3. Oliveira C, Barros G, Oliveira L. Estudo comparativo da radiopacidade entre três cimentos obturadores do sistema de canais radiculares. An Fac de Odontol UFPE 2001 11(1-2):22-8.

4. Cohen S, Burns R. Caminhos da polpa. 7 ed. Rio de Janeiro: Guanabara Koogan; 2000.

5. Siqueira JF, Jr., Favieri A, Gahyva SM, Moraes SR, Lima KC, Lopes HP. Antimicrobial activity and flow rate of newer and established root canal sealers. J Endod 2000 May;26(5):274-7.

6. Estrela C. Ciência endodôntica. São Paulo: Artes Médicas; 2004.

7. ASSOCIATION AD. Specification n. 57 for endodontic filling materials. Washington: ADA; 1983; Available from: http://www.ada.org/830.aspx.

8. Savioli R, Silva R, Pécora J. Influência de cada componente do cimento de Grossman sobre as propriedades físicas de escoamento. Tempo de endurecimento e espessura do filme. Rev Paulista de Odontol 1994 mai-jun.;16(3):14-6.

9. Guimarães C, Sampaio J, Sato E, Collesi R. Avaliação do escoamento de seis cimentos endodônticos. Rev Odontol Univ Santo Amaro 1999 jan-jun.;4(1):10.

10. Elias P, Costa R. Estudo da radiopacidade dos cimentos endodônticos Endofill e Sealer 26. Pesq Bras Odontoped Clin Integr 2003 jan-jun.;3(1):35-40.

11. Costa R, Scelza M, Costa A. Radiopacidade de cimentos endodônticos: avaliação da intensidade pixel. J Bras Clin Odontol Int 2002 mar-abr.;6(32):137-139.

12. Baksi BG, Sen BH, Eyuboglu TF. Differences in aluminum equivalent values of endodontic sealers: conventional versus digital radiography. J Endod 2008 Sep;34(9):1101-4.

REVISTA DE

ODONTOLOGIA DA

UNIVERSI DADE

CIDADE DE SÃO

PAULO

2011; 23(1): 15 -

22 , JAN-ABR
13. Tasdemir T, Yesilyurt C, Yildirim T, Er K. Evaluation of the radiopacity of new root canal paste/sealers by digital radiography. J Endod 2008 Nov;34(11):1388-90.

Recebido em: 23/02/2010

Aceito em: 9/08/2010 